\title{
Real-Time Driver Drowsiness Detection using Computer Vision
}

\author{
Mahek Jain, Bhavya Bhagerathi, Sowmyarani C N
}

\begin{abstract}
The proposed system aims to lessen the number of accidents that occur due to drivers' drowsiness and fatigue, which will in turn increase transportation safety. This is becoming a common reason for accidents in recent times. Several faces and body gestures are considered such as signs of drowsiness and fatigue in drivers, including tiredness in eyes and yawning. These features are an indication that the driver's condition is improper. EAR (Eye Aspect Ratio) computes the ratio of distances between the horizontal and vertical eye landmarks which is required for detection of drowsiness. For the purpose of yawn detection, a YAWN value is calculated using the distance between the lower lip and the upper lip, and the distance will be compared against a threshold value. We have deployed an eSpeak module (text to speech synthesizer) which is used for giving appropriate voice alerts when the driver is feeling drowsy or is yawning. The proposed system is designed to decrease the rate of accidents and to contribute to the technology with the goal to prevent fatalities caused due to road accidents.
\end{abstract}

Keywords: Drowsiness, eSpeak module, Eye aspect ratio, Yawn Detection.

\section{INTRODUCTION}

$\mathrm{D}$ river drowsiness and fatigue are one of the most common reasons for accidents. The number of fatalities due to such accidents is increasing worldwide each year. This paper aims to lessen the number of accidents due to driver drowsiness and fatigue. This will in turn increase transportation safety. Driver drowsiness detection is a technology in vehicles that is useful in preventing accidents and saving the lives of drivers when they are getting drowsy. This project uses computer vision for the detection of drivers' drowsiness. With the constant improvement and novelty in technology, there is an advancement in transportation modes. Our dependencies on it have started increasing at a high rate. It has greatly affected our lives in many ways. Considering any social status, there are some rules which should be followed by any vehicle driver. One is to stay alert and the other one is being active while driving. The existing technologies to detect driver drowsiness are either very costly systems that apply to the high-end car models or systems that are affordable but are not robust.

Manuscript received on September 22, 2021.

Revised Manuscript received on September 27, 2021.

Manuscript published on October 30, 2021.

*Correspondence Author

Mahek Jain*, Department of Computer Science and Engineering, Rashtreeya Vidyalaya College, Bangalore (Karnataka), India.

Bhavya Bhagerathi, Department of Computer Science and Engineering, Rashtreeya Vidyalaya College, Bangalore (Karnataka), India.

Dr. Sowmyarani C N, Associate Professor, Department of Computer Science and Engineering. Bengaluru (Karnataka), India.

(C) The Authors. Published by Blue Eyes Intelligence Engineering and Sciences Publication (BEIESP). This is an open access article under the CC BY-NC-ND license (http://creativecommons.org/licenses/by-nc-nd/4.0/)
This paper is focused on designing a drowsiness detection system that functions efficiently and is affordable. The method that is needed in the present scenario detects drowsiness based on the eyes and mouth geometric features. This paper proposes to achieve the same, by developing a drowsiness detection system to monitor and prevent a destructive outcome from the negligence of fatigue. There are an increasing number of accidents on roads today and driver drowsiness is an important factor and this has been accepted extensively. The exact number of accidents caused by driver's drowsiness is difficult to decide because it is underestimated most of the time. The transition from being fatigued to dozing off is subtle and generally goes unnoticed by the driver. This explains why it is important to do more work in this field with an aim to reduce the occurrence of accidents related to drowsiness and motivate ourselves to develop a driver drowsiness detection system.

\section{RELATED WORK}

The survey which is done includes the present technologies and research available related to the topic of our project. It is an attempt to better understand the efforts that have gone into this field of study, and also to understand where our efforts should be focused on while developing this project. This literature review has been carried out on the topic of the current drowsiness detection technologies for facial landmark detection [7], blink detection, and yawn detection. across many techniques to carry out drowsiness detection, which includes deep CNN [13], Computer Vision [15], Behavioral measures, and machine learning techniques all with different advantages, challenges, and different levels of accuracy. Research has been done on EAR and MAR-based technologies for blink detection and yawn detection respectively. From [1] titled "Computer Vision based drowsiness detection for motorized vehicles with Web Push Notifications". In this paper, they have designed a drowsiness system that is based on computer vision for vehicles with alarm sounds and web push notifications. These notifications will alert the driver to prevent the accident from occurring. The system is also designed to generate an alert that shows nearby coffee shops which will increase the alertness of the driver. As a result, the system detected the driver's drowsiness and showed successful performance in the trial run. The Eye Aspect Ratio was used to detect if the eyes are closed or open. Then, a buzzer was used to generate an alert and a web push notification was sent to the user which shows the nearby coffee shops.

Published By:

Blue Eyes Intelligence Engineering and Sciences Publication (BEIESP)

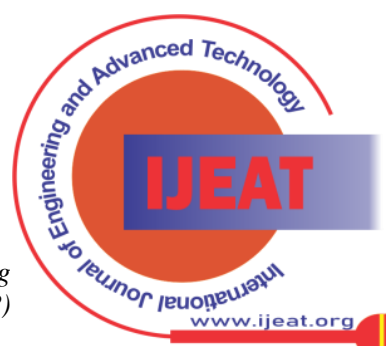

Exploring Innovation 
The limitations of the paper are that the Raspberry Pi camera was used and because of this, the system could not work during the nighttime. A night-vision camera should have been used. From [2] titled "Real-time monitoring of driver drowsiness on mobile platforms using 3D neural networks". In this paper, they have used depth-wise separable $3 \mathrm{D}$ convolution operations to detect drowsiness in drivers from real-time face video and also, they have identified micro sleeps and alerted the drivers. The results obtained show that the method can decide which features are important and it does not depend on the developer to pre-specify a set of features because they might miss some features like nose wrinkles, eyelid movement, and other facial gestures. The limitations of the paper are that the dataset which is used consists of just 18 persons and also, the frames were not labeled properly.

From [3] titled "The detection of drowsiness using a driver monitoring system". In this paper, they have made use of a driver monitoring system (DMS) to detect drowsiness along with different kinds of sensors. They have also collected data in the form of signals from other vehicle-based sensors. The results obtained show that the models were effective at dividing the drowsiness into three levels - low, moderate, and severe drowsiness. But, while differentiating between moderate and severe levels, the model was not efficient enough. The limitations of the paper are that the model was not effective while differentiating moderate drowsiness from severe drowsiness. Another limitation is that the size of the sample used in this paper is small.

From [4] titled "Driver Drowsiness Detection System Using Computer Vision”. The objective of the paper is to detect driver drowsiness by analyzing human eye blinks using a recent facial landmark detection and to make use of E.A.R(eye aspect ratio) for easy, fast, and efficient blink detection. The results showed that the system was successful in driver drowsiness detection by providing a reliably precise enough estimation of the level of eye openness. This alert system can be used in real-time due to a very negligible performance cost experienced in facial landmark detection. The limitations of the paper are that a fixed blink duration is assumed even though everyone's blink duration lasts differently. EAR is estimated from two-dimensional data which cannot account for out-of-plane head orientation and the model depends only on eyes for drowsiness detection.

From [5] titled "Drowsiness Detection Based on Eye Closure and Yawning Detection". In this paper, driver's eye and mouth movements are tracked using Haar-cascade classifiers. This will help to detect the closing of eyes and frequency of yawning. The system also generates alarm sounds if the driver is drowsy or already asleep. As a result, in $85 \%$ of the cases, the system detects faces and facial features which are required, accurately. The system is prompt in detecting drowsiness once the feature detection of the face is positive. The limitation of the paper is that it is observed that the system's accuracy decreases in bad lighting conditions.

\section{OBJECTIVES}

The main objective is to develop a system that is accurate to detect a driver's drowsiness based on eyelid movement and yawning and is reliable to give appropriate voice alerts [6] in real-time. The other objectives include designing a system that detects drowsiness of drivers by monitoring the eyes of the driver regularly, especially the retina. The system should give an alert to the driver when the driver yawns frequently or when the driver's eyes remain closed for a few seconds. The system works even when a driver is wearing spectacles. The system is not affected by bad lighting conditions.

\section{METHODOLOGY}

Firstly, the face is localized in the image using facial landmark detection. Then, shape prediction methods are used to detect important features on the face. Face detection [14] is done by OpenCV built in HAAR cascades, which are pre-trained. In the next step, to estimate the location of 68 (x, y)-coordinates that map to facial structures, a facial landmark detector which is pre-trained and included in the dlib library is used. It is also trained on the iBUG $300-\mathrm{W}$ dataset. The EAR [8] is computed using the ratio of distances between the horizontal and vertical eye landmarks for drowsiness detection. For the purpose of yawn detection [9], a YAWN value will be calculated using the distance between the upper and the lower lip, and the distance will be compared against a threshold value. eSpeak module (text to speech synthesizer) is used for giving appropriate voice alerts when the driver is feeling drowsy or is yawning.

\section{SOFTWARE REQUIREMENTS SPECIFICATION}

The proposed system must be able to detect drowsiness given a proper real-time driving environment. The performance will depend upon the quality of the camera as well. The proposed system due to its well-designed and easy-to-use interface can be used by both day-time and night-time drivers. Users can follow up the interface step by step for their purpose. The proposed system must be available for use to the user as and when needed provided that the user's system meets the specified requirements. The proposed system must be able to recover from failure in case of the application crashing abruptly and become ready-to-use after recovery. The prototype of the drowsiness detection system will be implemented on the Raspberry Pi microcontroller board, along with the necessary peripheral hardware, and Python 3 will be used to implement the software functionality of drowsiness detection.

\section{SYSTEM DESIGN}

\section{A. System Architecture}

When the driver is driving, the driver's face is captured by a camera and it is converted into a video stream. The application then analyzes the video to detect drowsiness and fatigue and also checks the level of drowsiness. In this stage, the main parts which should be considered for analysis are: the driver's face tracking, driver's fatigue state, and recognition of key regions of the face based on eye closure and yawning [10]. Finally, if the drowsiness is detected, a warning voice alert is given.

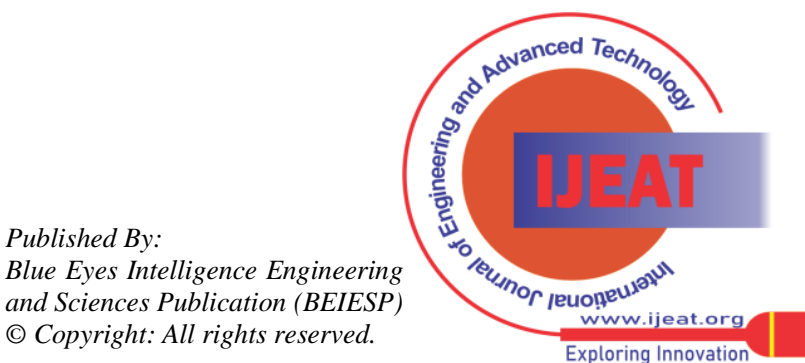


Fig. 1 depicts High-Level System Architecture consisting of the input to the model and the preprocessing and evaluation in stages. Stage 1 involves pre-processing of video stream for human face tracking. Stage 2 involves extraction of facial key regions such as eyes and mouth. Stage 3 involves detection of drowsiness symptoms like eye closure, blinking, and yawning.

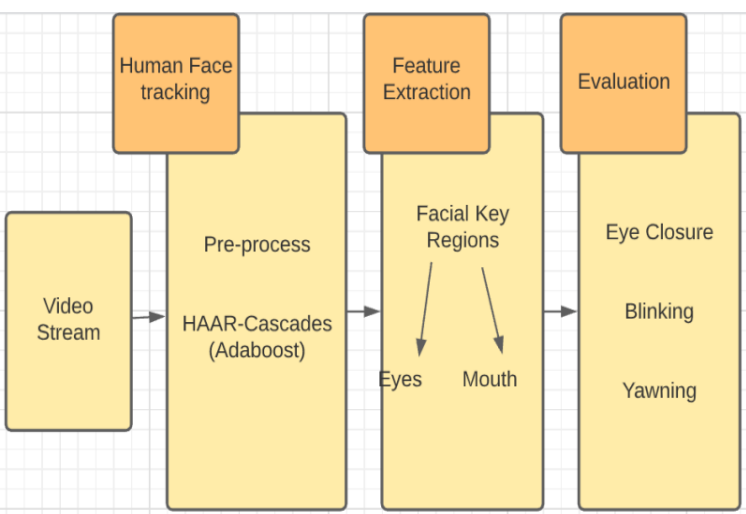

Fig. 1. High-Level System Architecture

\section{B. Detailed Design}

The system has been designed such that the face and hence the eyes and mouth of the driver are always monitored and if the predefined levels of alertness are observed to be defaulted and compromised, then an appropriate alarm is set off, and accordingly, action is taken to prevent any fatalities. Fig. 2 depicts the System Design of Driver Drowsiness and Yawn Detection System. It can be seen that the camera is used for monitoring the driver's face continuously and upon detection of drowsiness or fatigue, the system in the dashboard generates a voice alert type warning to the driver.

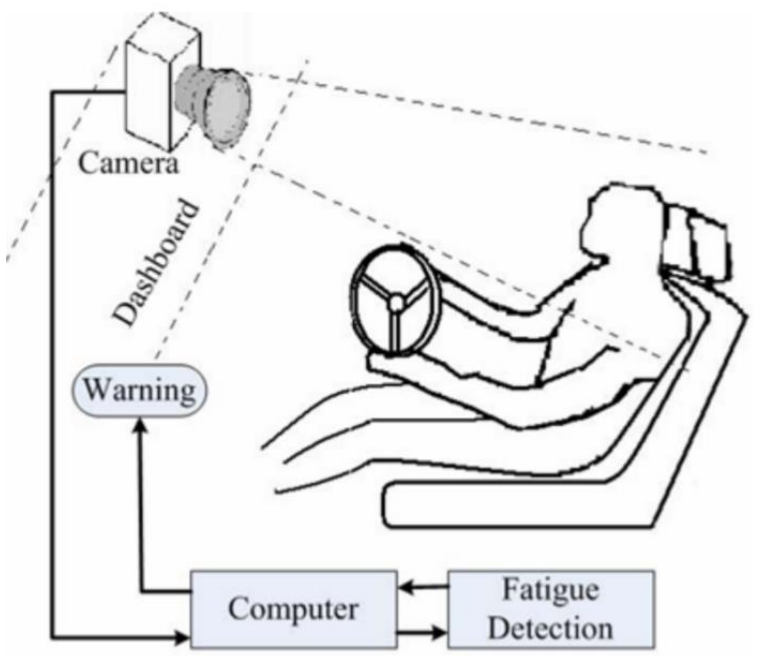

Fig. 2. System Design

\section{EXPERIMENTAL RESULTS}

\section{A. Experimental Dataset}

The experimental dataset used in our project is the iBUG-300w Dataset, which consists of 300 indoor and as many outdoor images. The dataset covers a large variety of identities, face size, lighting conditions, pose, etc. The images in the 300-W dataset cover more expressions than the common neutral smile such as surprise or scream. Annotations on the image were done using 68-point markup covering all the key facial landmarks including eyes, mouth, nose, etc. [19] But with regards to this project our region of interest (ROI) includes only the eyes and lips region which can be extracted using python slicing techniques.

Table- I: iBUG 300w Dataset split up

\begin{tabular}{|c|c|}
\hline Total Images & $\begin{array}{c}\text { Number of Annotated faces } \\
\text { involved }\end{array}$ \\
\hline 293 & 1 \\
\hline 53 & 2 \\
\hline 53 & {$[3,7]$} \\
\hline
\end{tabular}

\section{B. Performance Analysis}

To achieve the expected results, a large number of pictures were taken and their accuracy in drowsiness (using EAR calculation) and yawn detection [11] was analyzed. Sample 1: Face improperly aligned

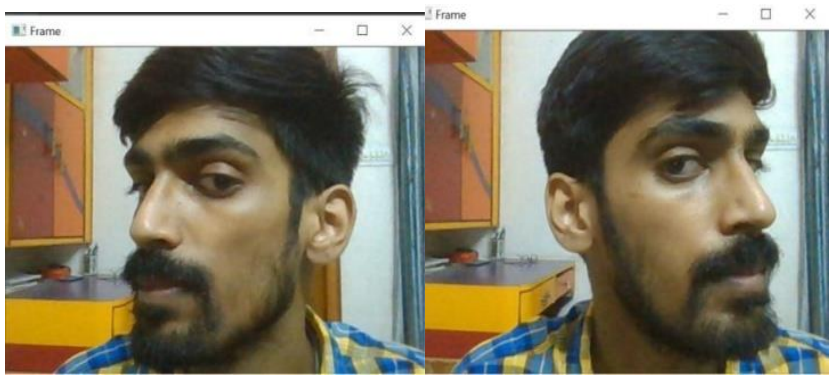

Fig. 3. Face improperly aligned

Sample 2: While wearing a hat (wearable obstacle)

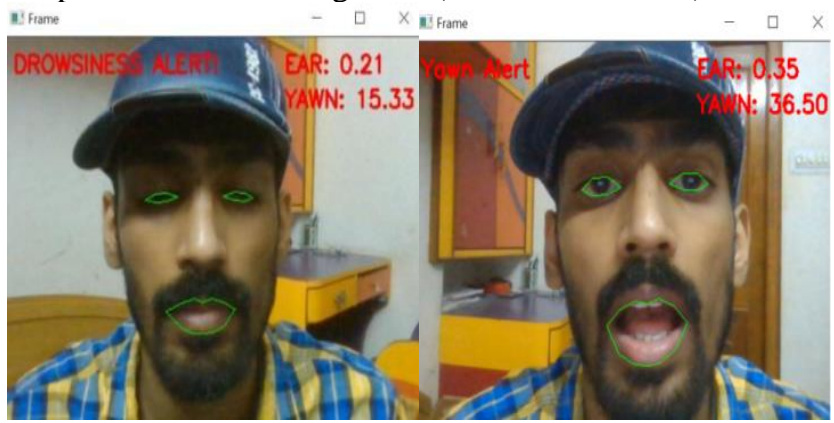

Fig. 4. Wearing obstacle (Hat)

Sample 3: Face properly aligned

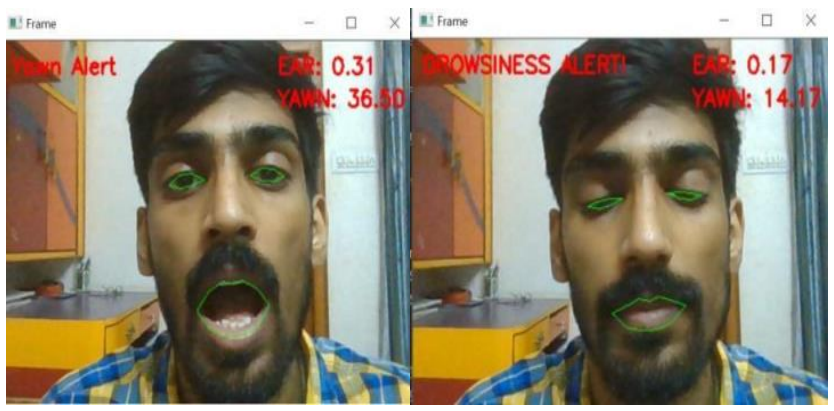

Fig. 5. Face properly aligned and eyes closed

Performance analysis of the system under different conditions is shown in the table below:

Published By:

Blue Eyes Intelligence Engineering and Sciences Publication (BEIESP) 


\section{Real-Time Driver Drowsiness Detection using Computer Vision}

Table- II: Performance Analysis

\begin{tabular}{|c|c|c|}
\hline I/P & $\begin{array}{c}\text { Drowsiness } \\
\text { Detection }\end{array}$ & Yawn Detection \\
\hline Sample 1 & $\begin{array}{c}\text { Not detected } \\
\text { (improper face } \\
\text { alignment) }\end{array}$ & $\begin{array}{c}\text { Not detected } \\
\text { (improper face } \\
\text { alignment) }\end{array}$ \\
\hline Sample 2 & $96 \%$ & $98 \%$ \\
\hline Sample 3 & $99 \%$ & $99 \%$ \\
\hline
\end{tabular}

The following parameters are considered for performance analysis:

- Drowsiness Detection Accuracy = total no. of times alert raised when eyes closed / (total no. of times alert raised when eyes closed + total no of times alert didn't raise when the eyes are closed)

- Yawn Detection Accuracy = total no. of times alarm raised when user yawns / (total no. of times alarm raised when user yawns + total no of times alarm didn't raise when the user yawns).

\section{System Testing}

The following table represents five test cases that were conducted while doing this project for drowsiness and yawn detection of the driver.

\section{Table- III: System Testing}

\begin{tabular}{|c|c|c|c|c|}
\hline Test Cases & $\begin{array}{c}\text { Eyes } \\
\text { Detected }\end{array}$ & $\begin{array}{c}\text { Eye } \\
\text { closure }\end{array}$ & $\begin{array}{c}\text { Yawn } \\
\text { detected }\end{array}$ & Result \\
\hline Case $\mathbf{1}$ & No & No & No & No result \\
\hline Case $\mathbf{2}$ & Yes & No & No & No result \\
\hline Case 3 & Yes & Yes & No & Voice alert \\
\hline Case 4 & Yes & No & Yes & Voice alert \\
\hline Case 5 & Yes & Yes & Yes & Voice alert \\
\hline
\end{tabular}

According to the methodology of the system, when the eyes are closed for more than the set threshold number of frames or when the driver is yawning [12], then the driver is feeling tired. Henceforth, one of these distinguished cases arises, and the corresponding result happens. The accuracy as measured in the performance analysis phase is almost found to be $100 \%$ in the case when the face is properly aligned and no wearable obstacle is present. Accuracy drops down a little when an obstacle is present (e.g., Hat). Ambient lighting conditions are very essential for getting proper results. In case, the user's eye closure and yawn occur simultaneously, a voice alert is raised but the system behaves in an erroneous and unsynchronized fashion. Hence, such a situation should be avoided to prevent any inconsistent results.

\section{CONCLUSION AND FUTURE SCOPE}

The model is capable of detecting drowsiness by monitoring the eyes and mouth. Shape prediction methods [16] are used to detect important features on the face. The inputs given to these methods are facial landmarks which are obtained from facial landmark detection. This module deals with the EAR function which computes the ratio of distances between the horizontal and vertical eye landmarks. An eSpeak module (text to speech synthesizer) is also deployed which is used for giving appropriate voice alerts when the driver is feeling drowsy or is yawning. The whole project is designed to decrease the rate of accidents and to contribute to the technology with the goal to prevent fatalities caused due to road accidents. The future work of this paper can be focused on the use of outer factors for measuring fatigue and drowsiness. [18] The outer factors may be weather conditions, state of the vehicle, time of sleeping and, mechanical data. Driver drowsiness is among the major threats to road safety, and in the case of commercial motor vehicle operators, the problem is particularly severe. The factors that contribute to this serious safety issue are twenty-four-hour services, unpredictable conditions of the environment, high annual mileage, and an increase of work schedules that are demanding. One important step of preventive measures that are needed to solve this problem is by continuously observing the driver's drowsiness state [17] and giving information about their state to the driver so that they can take necessary action. Presently, no adjustment can be done concerning the zoom or direction of the camera during the system operation. [20] In the future, more work can be done to automate the zoom on the eyes after they are localized.

\section{LIMITATIONS}

The accuracy of the model degrades if the eye frames are not captured clearly due to any kind of obstacles such as goggles or spectacles having reflection). Camera operations such as auto adjustments with respect to zoom and rotation are not considered in conducting experiments. Once the eyes are localized, zooming in automatically will help increase the accuracy. The accuracy of detection of eyes and mouth reduces when the driver is not facing the camera.

\section{ACKNOWLEDGMENT}

We thank Anirudh Devpura for his assistance in checking the case results of the experiment. We thank the professors of RV College and Engineering for their advice and suggestions.

\section{REFERENCES}

1. Rahul Atul Bhope, "Computer Vision based drowsiness detection for motorized vehicles with Web Push Notifications", IEEE 4th International Conference on Internet of Things, IEEE, Ghaziabad, India, 2019.

2. Jasper S. Wijnands, Jason Thompson, Kerry A. Nice, Gideon D. P, Aschwanden \& Mark Stevenson, "Real-time monitoring of driver drowsiness on mobile platforms using 3D neural networks", Neural Computing and Applications, 2019.

3. Chris Schwarz, John Gaspar, Thomas Miller \& Reza Yousefian, "The detection of drowsiness using a driver monitoring system", in Journal of Traffic Injury Prevention (Taylor and Francis Online), 2019.

4. Aditya Ranjan, Karan Vyas, Sujay Ghadge, Siddharth Patel, Suvarna Sanjay Pawar, "Driver Drowsiness Detection System Using Computer Vision.", in International Research Journal of Engineering and Technology(IRJET), 2020.

5. B.Mohana, C.M.Sheela Rani, "Drowsiness Detection Based on Eye Closure and Yawning Detection", in International Research Journal of Engineering and Technology(IRJET), 2019.

6. Driver Alert Control (DAC). (2016, Feb 10). Retrieved from http://support.volvocars.com/uk/cars/Pages/owners-manual. aspx?mc $=$ Y555\&my $=2015 \& s w=14 w 20 \&$ article $=2 e 82 \mathrm{f} 6 \mathrm{fc} 0$ d1139c2c0a801e800329d4e

7. Z. Mardi, S. N. Ashtiani, and M. Mikaili, "EEG-based drowsiness detection for safe driving using chaotic features and statistical tests," Journal of Medical Signals and Sensors, vol. 1, pp. 130-137, 2011.

8. T. Danisman, I.M. Bilasco, C. Djeraba and N. Ihaddadene, "Drowsy driver detection system using eye blink patterns," Universite Lille 1 \& Telecom Lille 1, Marconi, France, 2010.

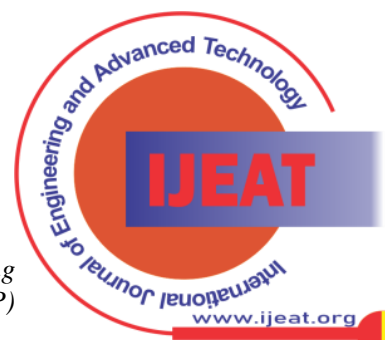


9. B. Hariri, S. Abtahi, S. Shirmohammadi, and L. Martel, "A yawning measurement method to detect driver drowsiness," Distributed and Collaborative Virtual Environments Research Laboratory, University of Ottawa, Ottawa, ON, Canada, 2011.

10. L. Li, Y. Chen and Z. Li, "Yawning detection for monitoring driver fatigue based on two cameras", Proceedings of the 12th International IEEE Conference Intelligent Transportation Systems., pp. 1-6, Oct. 2009.

11. S. Abtahi, B. Hariri and S. Shirmohammadi, "Driver drowsiness monitoring based on yawning detection", Proceedings of the IEEE International Control, Measurement and Instrumentation (CMI), IEEE, pp. 1-4, May 2011.

12. X. Fan, B. Yin, and Y. Sun, "Yawning detection for monitoring driver fatigue", Proceedings of the International Conference on Machine Learning and Cybernet, vol. 2, pp. 664-668, Aug. 2007.

13. F. N. Iandola, S. Han, M. W. Moskewicz, K. Ashraf, W. J. Dally and K. Keutzer, "Squeezenet: Alexnet-level accuracy with 50x fewer parameters and $<0.5 \mathrm{Mb}$ model size", Proceedings of the International Conference of Learning Representations, pp. 1-13, 2017.

14. K. Zhang, Z. Zhang, Z. Li and Y. Qiao, "Joint face detection and alignment using multitask cascaded convolutional networks", IEEE Signal Processing Letters, vol. 23, no. 10, pp. 1499-1503, Oct. 2016.

15. D. B. Lucas and T. Kanade, "An iterative image registration technique with an application to stereo vision", Proceedings of the 7th International Joint Conference on Artificial Intelligence, vol. 2, pp. 674-679, 1981.

16. D. S. Bolme, J. R. Beveridge, B. A. Draper and Y. M. Lui, "Visual object tracking using adaptive correlation filters", Proceedings of the IEEE Computer Society Conference on Computer Vision Pattern Recognition., pp. 2544-2550, Jun. 2010.

17. F. J. Henriques, R. Caseiro, P. Martins and J. Batista, "Exploiting the circulant structure of tracking-by-detection with kernels", Proceedings of the European Conference Computer Vision, pp. 702-715, 2012.

18. Y. Li and J. Zhu, "A scale adaptive kernel correlation filter tracker with feature integration", Proceedings of the European Conference Computer Vision, pp. 254-265, 2015.

19. M. Danelljan, G. Häger, F. S. Khan and M. Felsberg, "Discriminative scale space tracking", IEEE Transactions on Pattern Analysis and Machine Intelligence, vol. 39, no. 8, pp. 1561-1575, Aug. 2017.

20. N. Wang and D.-Y. Yeung, "Learning a deep compact image representation for visual tracking", Proceedings of the 26th International Conference on Neural Information Processing Systems., vol. 1, pp. 809-817, 2013.

\section{AUTHORS PROFILE}

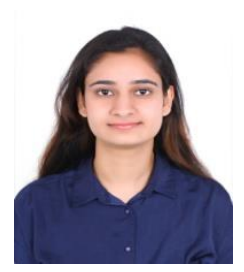

Mahek Jain, is currently a final year undergraduate student in the Computer Science and Engineering department at Rashtreeya Vidyalaya College of Engineering, Bangalore, Karnataka, India. She is an IT head and member of Ashwa Racing student organization at RVCE, which builds racing cars and competes at global level competitions. Her current research interests include applying deep learning algorithms in autonomous vehicle modules for pattern recognition and computer vision problems.

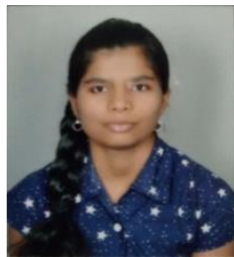

Bhavya Bhagerathi, is currently a final year undergraduate student in the Computer Science and Engineering department at Rashtreeya Vidyalaya College of Engineering, Bangalore, Karnataka, India. Her research interests include computer vision, image processing, image analysis and intelligent systems. She has actively taken part in research internships and intermediate-level projects. Notably, her recent works have focused on projects related to computer vision and image segmentation using machine learning.

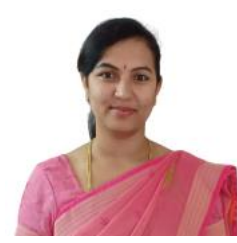

Dr. Sowmyarani $\mathbf{C} \mathbf{N}$, is currently working in Rashtreeya Vidyalaya College of Engineering as an Associate Professor in the Department of Computer Science and Engineering. She has secured her M.E. degree from UVCE, Bengaluru with 2nd rank in university and secured her Ph.D. degree from VTU, Belagavi. She has published more than forty international Journals Conference papers and book chapters. She is an active member of the IEEE association, ISTE, and ACM Her areas of interest are Data Privacy, Computer Networks, Smart technologies, and Education Technology.

Published By:

Blue Eyes Intelligence Engineering and Sciences Publication (BEIESP) (c) Copyright: All rights reserved.

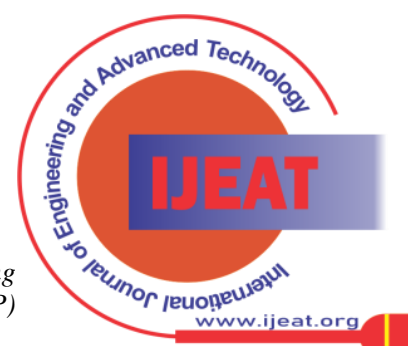

Exploring Innovation 